\title{
Frozen elephant trunk for DeBakey type 1 dissection: the Cleveland Clinic technique
}

\author{
Eric E. Roselli, Michael Z. Tong, Faisal G. Bakaeen \\ Aorta Center, Department of Thoracic and Cardiovascular Surgery, Heart and Vascular Institute, Cleveland Clinic, Cleveland, Ohio, USA \\ Correspondence to: Eric E. Roselli, MD. Cleveland Clinic, Department of Thoracic and Cardiovascular Surgery, 9500 Euclid Avenue/Desk J4-1, \\ Cleveland, Ohio 44195, USA. Email: roselle@ccf.org.
}

Submitted Mar 10, 2016. Accepted for publication May 09, 2016.

doi: 10.21037/acs.2016.05.03

View this article at: http://dx.doi.org/10.21037/acs.2016.05.03

\section{Clinical vignette}

A 67-year-old male presented with abrupt onset of chest and back pain to an outside hospital. He was hypertensive with a serum creatinine of $4.4 \mathrm{~g} / \mathrm{dL}$ and reported occasional cocaine use. Comorbidities include chronic renal insufficiency (baseline serum creatinine $1.8 \mathrm{~g} / \mathrm{dL}$ ), morbid obesity [body mass index (BMI) of 38], history of atrial fibrillation, and status post permanent pacemaker. Computed tomography (CT) demonstrated DeBakey type 1 dissection. The referral was received, and the Cleveland Clinic Acute Aortic Syndrome team was activated. The emergency medical transport team delivered the patient to our main campus where anti-impulse therapy was optimized in the cardiovascular triage intensive care unit and CT images were reviewed. CT demonstrated an extensive aortic dissection with poor visualization of the entry tear and a maximum descending aortic diameter of $40 \mathrm{~mm}$ just beyond the left subclavian artery. Also of interest, this patient had an anomalous left vertebral artery originating directly from the aortic arch distal to the origin of the left subclavian artery. He was taken to the hybrid operating room for emergency ascending and modified simplified branched frozen elephant trunk repair.

\section{Surgical techniques}

\section{Patient selection}

For patients with acute ascending dissection extending beyond the aortic arch (DeBakey type 1), we frequently perform an extended repair using a simplified frozen elephant trunk strategy, including ascending \pm root replacement with a surgical graft, plus total arch and proximal descending repair with direct placement of a thoracic stentgraft device sutured into the arch and directly to the proximal surgical graft $(1,2)$. This technique can be used for any patient with type 1 dissection but is an especially helpful technique for patients with retrograde dissection from a distal entry tear, aneurysmal changes in the more distal aorta, distal malperfusion, and those with a connective tissue disorder or family history placing them at increased risk for false lumen aneurysm of the distal aorta (3).

This surgical technique has evolved over the last 6 years, and the latest iteration will be presented in the accompanying video (Video 1).

\section{Preparation}

The Acute Aortic Syndrome team consists of emergency care transport, cardiology intensive care physicians (cardiology), cardiovascular imaging (radiologists/cardiologists), cardiothoracic anesthesia, cardiovascular surgery, vascular surgery, operating room nursing, radiology technicians, and perfusion. All members of this team are notified when a possible aortic dissection is en route (4).

Careful review of contrast enhanced three-dimensional CT imaging is required by the operating surgeon to understand the details of the patient's anatomy, the morphology of the dissection, and to size the devices used during the repair. The stent graft is sized based on the diameter of the aorta in cross section to the centerline of flow at the level of the left common carotid artery without oversizing (Figure 1). If branch vessel stent grafting is also planned, these devices are also pre-selected based on analysis of the three dimensional reconstructions with minimal oversizing. For the main aortic graft, the length 
A

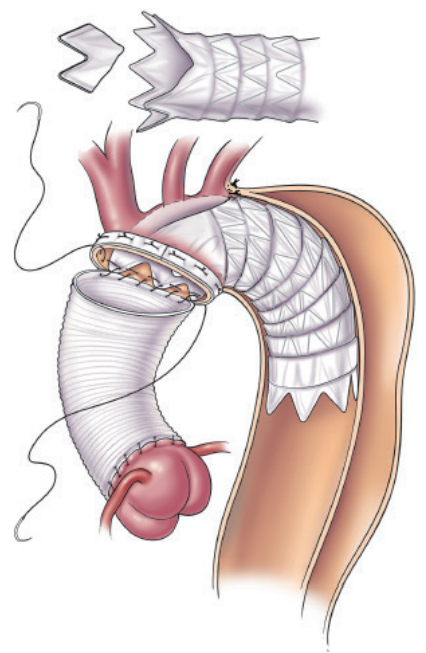

B

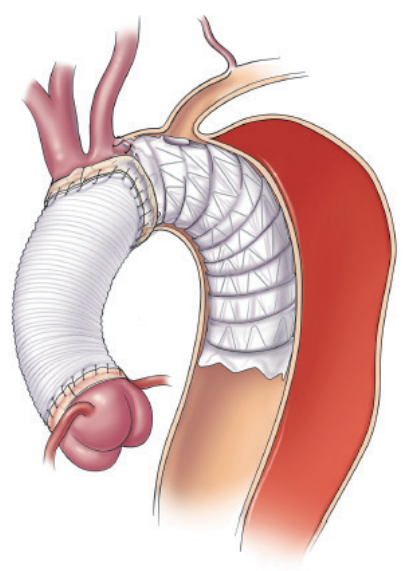

C

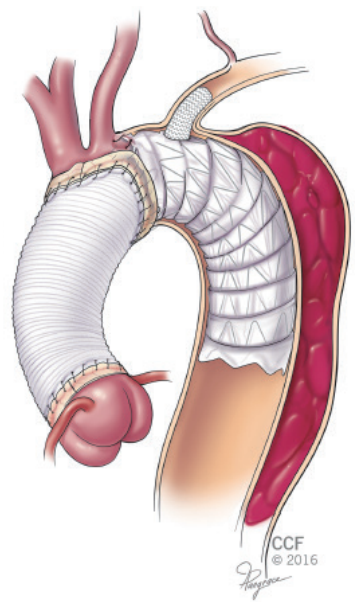

Figure 1 Evolution of the simplified frozen elephant trunk repair technique for acute aortic dissection. (A) Circa 2009 with scallop creation (B) circa 2012 with on table fenestration; and (C) circa 2015 with direct arch branch stentgrafting.

of the device is either 10 or $15 \mathrm{~cm}$ depending on the shape of the arch, so that it extends around the curve of the arch and into the upper descending aorta. For the branched graft devices, the $2.5 \mathrm{~cm}$ length is preferred.

To expedite repair, we typically forego preoperative cardiac catheterization unless the patient has a history of previous cardiac surgery and known coronary disease. Transesophageal echocardiography (TEE) is performed in the operating room to assess valve function. All patients receive broad-spectrum intravenous antibiotics with both gram positive and gram negative coverage. Cerebral monitoring is facilitated by the use of near infrared spectroscopy as all patients undergo hypothermic circulatory arrest with selective antegrade brain perfusion (SABP).

\section{Exposure}

Right axillary cannulation is preferred for arterial inflow during cardiopulmonary bypass and this facilitates the use of SABP. Approach to the aorta is through a median sternotomy. In the event that the patient becomes unstable during exposure of the right axillary artery, sternotomy is performed immediately to relieve tamponade and the patient's status is reassessed. If the patient is stabilized after relief of tamponade, then we proceed with right axillary cannulation; if not, then we move to central aortic cannulation using a modified Seldinger technique guided by TEE. Venous cannulation is usually performed using a multi-stage cannula in the right atrium with or without an additional cannula in the superior vena cava to augment drainage of the head. The coronary sinus is cannulated for the delivery of retrograde cardioplegia.

Cooling begins once cardiopulmonary bypass is instituted to a goal blood temperature of $18-20^{\circ} \mathrm{C}$, and the head is packed in ice. Bispectral index (BIS) monitor is used to confirm adequate cooling and cerebral oximetry is used to confirm adequate bilateral flow during SABP. After at least twenty minutes of cooling, we look for BIS silence and a bladder temperature of at least $28{ }^{\circ} \mathrm{C}$ before switching to partial cardiopulmonary bypass with SABP. The SABP is run at a rate of $10-12 \mathrm{cc} / \mathrm{kg} /$ minute at a perfusate temperature of $<18^{\circ} \mathrm{C}$.

Although we initially used transfemoral wire access for all of the frozen elephant trunk cases, we now reserve it for cases with particularly complicated anatomy (e.g., very tortuous arch, coarctation, previous descending repair). The stentgraft device for performing the frozen elephant trunk is sized based on the level of the aorta at the junction between the left common carotid and left subclavian arteries. It is important that this measurement is made using threedimensional reconstruction software so that the diameter is in a plane orthogonal to the center line of flow. The device is not oversized because the proximal end will be sutured directly to the aortic wall (so migration is not an issue) and in the decompressed state the stentgraft device is easier to sew to with good sealing if it is not folded upon itself within the depressurized aorta. The length of the stentgraft is typically $10-15 \mathrm{~cm}$, and is selected so that the distance from 
the proximal arch point of fixation to the end of the device is sufficient to get around the curved portion of the arch and into the proximal straight segment of the descending aorta. For a tight arch, $10 \mathrm{~cm}$ is often long enough and for an unfolded arch, $15 \mathrm{~cm}$ may be necessary. Use of a device longer than that may put the patient's spinal cord at unnecessary risk.

\section{Operation}

After safe sternal entry and pericardiotomy, the patient is cannulated as described above. Cardiopulmonary bypass is initiated and cooling is begun. A mobile self-retaining retractor attached to the sternal retractor (Hercules; Estech, West Chester, OH, USA) lifts the innominate vein upward and away from the supra-aortic branch vessels. During the cooling phase, the proximal arch is more fully exposed by taking down the pericardial reflection and encircling the innominate and left common carotid arteries. Once the patient achieves an adequate level of cooling, SABP is started by clamping the innominate and left common carotid arteries. The proximal aorta is then opened. The arch is transected obliquely from the base of the innominate artery to the underside of the aortic arch as is commonly performed for a conventional hemiarch aortic repair. To avoid steal from the left vertebral system, a 9-Fr occlusion balloon may be placed into the left subclavian artery.

The stentgraft selected for the frozen elephant trunk is delivered across the aortic arch and into the proximal descending within the true lumen of the aorta. To facilitate placement of the stentgraft device into the dissected descending aorta, it is pre-curved. When positioning the device in the aorta it is important not to force it into position and to visualize it directly throughout its course into the aorta. After the device is deployed within the true lumen, its exact position may be manually adjusted. It is sutured to the base of the lesser curve of the aortic arch with a 4-0 polypropylene suture. Along the greater curve, the device is pulled forward to lie proximally within the aortic arch. It is usually positioned so that it covers the left subclavian artery and sometimes it may cover the left common carotid artery too. A handheld eyeball cautery is then used to create a fenestration in the device directly under the left subclavian artery and any other branch arteries too if they are covered. The case demonstrated in the accompanying video had coverage of both the left subclavian artery and the anomalous left vertebral artery that originated directly from the aortic arch.
Although an iteration of this technique was first described in 2012, it has gone through some modifications through the last several years (1). Initially it was performed by resecting a portion of the flares on the first generation TAG stentgraft (Gore, Flagstaff, AZ, USA). Since that device has been replaced by one without flares, the procedure then evolved to include the creation of branch specific fenestrations as described here, and this has been performed with several of the commercially available stent grafts. In some patients with dissection extending into the branch vessels, we had noticed that there was persistent flow into the false lumen from these dissected branch communications around the stentgraft device. The procedure has since undergone additional modifications to become an operation that also may include direct placement of branch stentgrafts through these fenestrations into the arch vessels as shown in the accompanying video (Video 1).

Like sizing for the main aortic device, sizing for the branch vessels is done with three dimensional analysis and minimal oversizing of the target vessel. Short branch stent grafts measuring approximately $2.5 \mathrm{~cm}$ are selected for this portion of the procedure. After creation of the fenestrations, the branch stentgrafts are placed directly through the fenestration into the target vessel and deployed. Positioning is confirmed manually and the devices are dilated open both by direct manipulation with a clamp and by balloon molding with the soft conformable 9 Fr occlusion balloon described earlier. The branch devices are aligned so that the first stent of the branch device extends into the aortic lumen. These devices may be sutured together if desired.

Once the device delivery and deployment has been completed, the main aortic stentgraft device is directly sutured to the transected aortic wall with a running polypropylene suture and supported with a strip of bovine pericardium on the outer wall. The surgical graft is selected based on the size of the sinotubular junction and beveled distally so that the shorter edge is aligned with the lesser curve of the aortic arch. The surgical graft is then sutured circumferentially to the transected aorta, the stentgraft, and the base of the innominate artery ( \pm left common carotid artery) with running polypropylene suture. After completion of that anastomosis, the distal aorta is de-aired and clamped proximally to allow for reinstitution of full cardiopulmonary bypass flow. After correction of metabolic derangements is confirmed, the rewarming process is resumed.

\section{Completion}

Attention is then returned to the proximal aortic reconstruction 
guided by the condition of the aortic valve and root. Most commonly, the valve and root are both preserved by reapproximation of the dissected layers at the level just above the coronary ostia, with re-suspension of the aortic valve commissures, and direct suturing of the proximal end of the surgical graft. This is typically done in two layers. A running 5-0 polypropylene suture is first run around the sinotubular junction and supported by a strip of bovine pericardium around the outer layer, and the second layer involves the anastomosis to the surgical graft. Other options included valve replacement with a bioprosthesis and supracoronary graft anastomosis, total root replacement with reimplantation of the coronaries and valve replacement (modified Bentall procedure), or root replacement with valve reimplantation (modified David's procedure in young stable patients).

If there is concern that the patient may have ongoing distal ischemia, then aortography or intravascular ultrasound may be performed and additional endovascular therapies directed at static branch occlusion can be performed expeditiously in the hybrid operating room.

Post-operative care proceeds as is standard for patients who present with acute aortic dissection. Patients are imaged with computed tomography before discharge and this is done with contrast if renal function allows for it. Subsequent follow-up CT imaging is performed at approximately three months postoperatively and then annually thereafter depending on the findings.

\section{Comments}

Surgical intervention for acute aortic dissection had not changed appreciably for over 20 years between the 1980s and the turn of the century. Nonetheless, acute outcomes continued to improve because the overall care of patients improved. More recent data have demonstrated that the experience of the center has a positive impact on outcomes. Patients who present with ischemia still represent a difficult subset of patients, and during intermediate followup, survival is still dependent upon the patency of the downstream false lumen $(5,6)$. The recent development of frozen elephant trunk procedures to extend repair beyond the arch shows potential to address some of these shortcomings of the conventional repair. Only a few centers have been early adopters of the frozen elephant trunk technique during the acute phase of aortic dissection because of concerns that the operation may be too complicated during an emergency (7).

\section{Clinical results}

In the initial report describing our use of this technique, 17 patients had the repair for acute aortic dissection. Technical success was achieved in all patients with no intraoperative deaths and no type 1 or 3 endoleaks. There was only one early death in an obese patient who had a massive pulmonary embolus on postoperative day 19 , and one late death due to pneumonia 18 months postoperatively. Clinical and CT imaging assessment was performed prior to discharge, within the first 6 and 12 months postoperatively, and annually thereafter. We have since performed this repair in 56 patients with acute dissection with similar results. Although some patients have required additional interventions, they have all been performed with an endovascular approach and no patients have required redo sternotomy and arch repair.

Since the video was first created for this patient, he returned to the emergency department with new back pain at about 7 weeks postoperatively. The patient's creatinine had returned to his baseline level of $1.7 \mathrm{~g} / \mathrm{dL}$. He underwent CT demonstrating healing of the false lumen around the stentgrafted segment of the frozen elephant trunk, but significant growth of the descending aorta beyond the repair by nearly one centimetre compared to the pre-discharge study. Fortunately, the patient was able to easily undergo thoracic endovascular aortic repair as an extension procedure using a percutaneous approach guided by intravascular ultrasound. If he had presented with early rapid growth after conventional repair, it would have required a very high risk operation, particularly with regard to his renal dysfunction.

\section{Advantages}

The single anastomosis technique described here allows for an extended repair while keeping the operation relatively simple. A single distal anastomosis much like what is done during conventional hemiarch repair is performed (Video 1). Use of this technique keeps the circulatory arrest period short, and the potential bleeding sites within close reach of the surgeon. The extended repair addressed the entire aortic arch and promotes remodeling of the aorta through the upper descending aorta. An added advantage of this approach is that it optimizes true lumen flow downstream and has the potential to reduce the risk of death in those who present with ischemia. 


\section{Caveats}

Choosing the proper size of the stentgraft is dependent upon CT imaging analysis and some of these preoperative studies are less than ideal given their emergency indications. The stentgraft devices are used in an off-label manner for this operation so not all operators will be comfortable with the described manipulations. Branch vessel dissection can pose a problem but the latest iteration of this technique utilizing branch stent grafting addresses this issue. Further evaluation of the currently described technique is needed. Hopefully, disease-specific devices will be developed that could address some of these limitations.

\section{Acknowledgements}

The authors wish to thank Joseph Pangrace for his artwork.

\section{Footnote}

Conflicts of Interest: Dr. Roselli is a consultant for Gore and Medtronic, and receives honoraria for speaking from Medtronic and Vascutek. The other authors have no conflicts of interest to declare.

\section{References}

1. Roselli EE, Rafael A, Soltesz EG, et al. Simplified frozen

Cite this article as: Roselli EE, Tong MZ, Bakaeen FG. Frozen elephant trunk for DeBakey type 1 dissection: the Cleveland Clinic technique. Ann Cardiothorac Surg 2016;5(3):251-255. doi: $10.21037 /$ acs.2016.05.03 elephant trunk repair for acute DeBakey type I dissection. J Thorac Cardiovasc Surg 2013;145:S197-201.

2. Roselli EE. Clearing the next hurdles in the treatment of acute ascending aortic syndrome. Eur J Cardiothorac Surg 2015;47:205-8.

3. Shrestha M, Bachet J, Bavaria J, et al. Current status and recommendations for use of the frozen elephant trunk technique: a position paper by the Vascular Domain of EACTS. Eur J Cardiothorac Surg 2015;47:759-69.

4. Schoenhagen P, Roselli EE, Harris CM, et al. Online network of subspecialty aortic disease experts: Impact of "cloud" technology on management of acute aortic emergencies. J Thorac Cardiovasc Surg 2016. [Epub ahead of print].

5. Geirsson A, Szeto WY, Pochettino A, et al. Significance of malperfusion syndromes prior to contemporary surgical repair for acute type A dissection: outcomes and need for additional revascularizations. Eur J Cardiothorac Surg 2007;32:255-62.

6. Fattouch K, Sampognaro R, Navarra E, et al. Longterm results after repair of type a acute aortic dissection according to false lumen patency. Ann Thorac Surg 2009;88:1244-50.

7. Girardi LN. Total arch for type A dissection? J Thorac Cardiovasc Surg 2016;151:294-5. 